University of Wollongong

Research Online

SMART Infrastructure Facility - Papers

Faculty of Engineering and Information

Sciences

2013

Reliability-Based Modeling of Park-and-Ride Service on Linear Travel Corridor

David Wang

Nanyang Technological University

Bo Du

University of Wollongong, bdu@uow.edu.au

Follow this and additional works at: https://ro.uow.edu.au/smartpapers

Part of the Engineering Commons, and the Physical Sciences and Mathematics Commons

Research Online is the open access institutional repository for the University of Wollongong. For further information contact the UOW Library: research-pubs@uow.edu.au 


\title{
Reliability-Based Modeling of Park-and-Ride Service on Linear Travel Corridor
}

\begin{abstract}
The modeling of multimodal choice in a railway-highway system with single park-and-ride service on a linear travel corridor is studied. Commuters choose either auto or railway to travel directly from home to city center or drive to the park-and-ride facility and transfer to railway transit service. Both the traffic congestion on the highway and the crowding on rail transit are considered. The highway capacity is assumed to be stochastic to take into account travel time reliability for use of the auto mode. Commuters are assumed to be distributed uniformly along the corridor. A linear complementarity system to model commuters' mode choice along the corridor and to solve the spatial equilibrium travel pattern is proposed. The formulated linear complementarity system is transformed into a mixed integer linear program to be solved. The modeling approach and solution algorithm are implemented in a small numerical example.
\end{abstract}

\section{Keywords}

reliability-based, corridor, linear, travel, service, park-and-ride, modeling

Disciplines

Engineering | Physical Sciences and Mathematics

\section{Publication Details}

Wang, D. Z. W. \& Du, B. (2013). Reliability-Based Modeling of Park-and-Ride Service on Linear Travel Corridor. Transportation Research Record, 2333 16-26. 


\title{
Reliability-Based Modeling of Park-and-Ride Service on Linear Travel Corridor
}

\author{
David Z. W. Wang and Bo Du
}

\begin{abstract}
The modeling of multimodal choice in a railway-highway system with single park-and-ride service on a linear travel corridor is studied. Commuters choose either auto or railway to travel directly from home to city center or drive to the park-and-ride facility and transfer to railway transit service. Both the traffic congestion on the highway and the crowding on rail transit are considered. The highway capacity is assumed to be stochastic to take into account travel time reliability for use of the auto mode. Commuters are assumed to be distributed uniformly along the corridor. A linear complementarity system to model commuters' mode choice along the corridor and to solve the spatial equilibrium travel pattern is proposed. The formulated linear complementarity system is transformed into a mixed integer linear program to be solved. The modeling approach and solution algorithm are implemented in a small numerical example.
\end{abstract}

As a useful travel demand management strategy, park-and-ride (P\&R) services have been widely used since the 1930s $(1,2)$ and have proved to be effective in reducing traffic congestion and air pollution in studies in the United Kingdom and Eastern Asia, for example, in Hong Kong and Singapore, especially for increased travel demand in the morning peak hours (3-6). The principle of $\mathrm{P} \& \mathrm{R}$ service is to encourage commuters to choose the combination of auto and public transit modes to reduce auto traffic in the central business district (CBD). The P\&R mode allows commuters to drive private cars to the $P \& R$ facilities and then to take public transit to their workplaces.

In previous studies, Garcia and Marin presented a bilevel model to find optimal parking investment and pricing decisions, and a simulated annealing algorithm was adopted to solve the model (7). Wang et al. formulated a multimodal user equilibrium model for the city in the morning peak hours to determine the optimal P\&R location and parking charge with the objectives of profit maximization and social cost minimization (8). Wang et al. considered a congested highway and a congestion-free railway to compare the characteristics of the mode choice equilibrium before and after introduction of a P\&R service and derived the optimal $P \& R$ locations and parking charges to maximize profit and minimize social cost (9). As an extension to the work by Wang et al. (9), Liu et al. relaxed two assumptions: the railway

School of Civil and Environmental Engineering, Nanyang Technological University, 50 Nanyang Avenue, Singapore 639798. Corresponding author: D. Z. W. Wang, wangzhiwei@ntu.edu.sg.

Transportation Research Record: Journal of the Transportation Research Board, No. 2333, Transportation Research Board of the National Academies, Washington, D.C., 2013, pp. 16-26.

DOI: $10.3141 / 2333-03$ is congestion-free and only one P\&R facility is considered (10). A crowding cost function was considered in the generalized cost of the railway, and infinite $\mathrm{P} \& \mathrm{R}$ services were provided along the corridor instead. A deterministic continuum equilibrium model was developed by applying an infinite mathematical programming approach to represent mode choice and $\mathrm{P} \& \mathrm{R}$ transfer behaviors, and the model was transformed to a discretized problem to be solved.

This study contributes to the literature in two major aspects. First, travel time reliability is considered in modeling the multimodal choice behavior. Empirical studies have suggested that travel time reliability plays an important role in travelers' route choice behavior (11). In this study, it is assumed that the highway has stochastic capacity, and therefore travel time is not deterministic but stochastic. Commuters using the highway have to reserve a time budget to make sure of not being late with a certain required level. Meanwhile, the travel time for rail transit services is assumed to be fixed. A higher level of travel time reliability is regarded as one of the advantages of rail transit use and taking this factor into account makes the model more realistic.

Second, a linear complementarity system (LCS) is adopted to formulate the multimodal choice behavior throughout the corridor. The LCS formulation belongs to the modeling paradigm class of differential variational inequalities (DVIs), which was introduced by Pang and Stewart (12). The multimodal choice at each home location is modeled into a complementarity problem, and the spatial interaction of the travel costs between different locations can be characterized by ordinary differential equations. Therefore, it is natural to apply the DVI paradigm to model the spatial equilibrium travel pattern with multimodal choice on a linear traffic corridor. In the literature, Liu et al. proposed an infinite mathematical programming method to formulate the similar problem of the spatial equilibrium travel pattern (10). Nevertheless, as is well recognized, the optimization formulation of user equilibrium is not applicable when the travel modes have nonseparable cost functions. Meanwhile, the DVI modeling approach applied in this study is more general and is able to accommodate the situation with modal-specific cost.

\section{CORRIDOR WITH MULTIMODAL CHOICE}

A corridor with $P \& R$ interchange service provides three travel modes: transit, auto, and $\mathrm{P} \& \mathrm{R}$ as schematically shown in Figure 1.

Let $L$ represent the length of the corridor and $Q$ denote the total number of commuters on this corridor. It is assumed that all commuters are continuously distributed along the corridor and the density of travel demand at location $x$ is $q_{0}(x)$, where $x$ is the distance from the location to the CBD. It is assumed that only one P\&R 


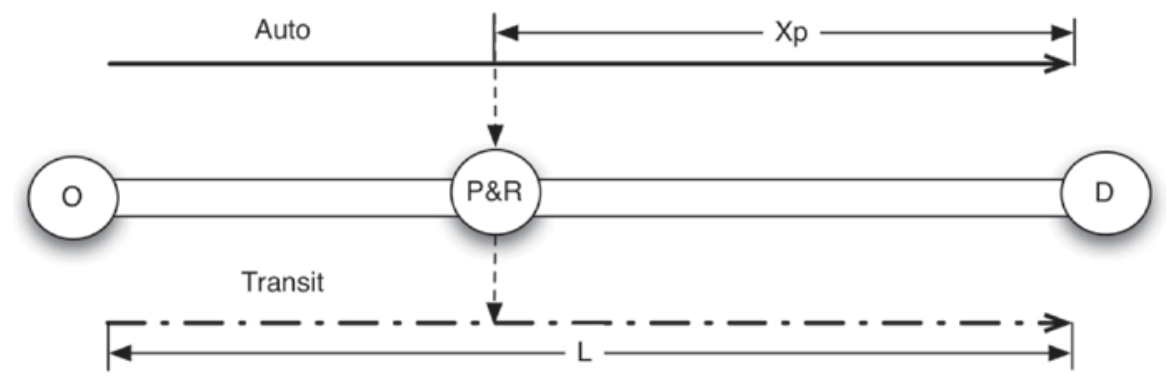

FIGURE 1 Corridor with multimodal choice.

facility is available on the corridor, with location $x_{p}$, as shown in Figure 1.

\section{Stochastic Link Capacity}

In this study, stochastic link capacity (SLC) is assumed for the highway. Considering that disruptions, such as traffic accidents, earthquakes, and so on, may occur on the transportation network, which result in reducing the effective link capacities and degrading the performance of the network, it is important to describe and quantify such impacts to improve the network design or develop incident management strategies (13). It is further assumed that the link capacity $C_{x}$ at location $x$ is the single source of uncertainty and that at each location the link capacity follows a specific probability distribution. For simplicity, a uniform distribution is adopted here for illustrative purposes. If the maximum capacity and minimum capacity are denoted $C_{\max }$ and $C_{\min }$, respectively, the uniform probability distribution function can be described as follows:

$f\left(C_{x}\right)=\frac{1}{C_{\max }-C_{\min }} \quad C_{x} \in\left[C_{\min }, C_{\max }\right]$

where $C_{\min }>0$ and $C_{\max }>0$.

Then the expectation and variance of the link capacity can be calculated as

$E\left(C_{x}\right)=\frac{C_{\max }+C_{\min }}{2}$

$D\left(C_{x}\right)=\frac{\left(C_{\max }-C_{\min }\right)^{2}}{12}$

where $C_{x}$ denotes the SLC at location $x$, whose probability distribution is assumed to be given exogenously.

Let $t_{h}\left(v_{h}(x)\right)$ represent the time to traverse a unit of distance at location $x$, where $v_{h}(x)$ is the traffic volume at location $x$. Then the travel time can be calculated by the Bureau of Public Roads function:

$t_{h}\left(v_{h}(x)\right)=t_{h}^{0}\left[1+A\left(\frac{v_{h}(x)}{C_{x}}\right)^{B}\right]$

where $t_{h}^{0}$ represents the free-flow travel time per unit of distance and $A$ and $B$ are calibrated parameters.

As described by D'Este, the travel time is supposed to be fully described by a first-order model where $B=1$ (14). The traffic volume at location $x$ on the highway is the accumulation of travelers choosing the auto mode and the $\mathrm{P} \& \mathrm{R}$ mode, if any, beyond location $x$, which can be described by

$v_{h}(x)= \begin{cases}\int_{x}^{L} q_{h}(y) d y & \text { if } x \in\left[0, x_{p}\right] \\ \int_{x}^{L}\left(q_{h}(y)+q_{p}(y)\right) d y & \text { if } x \in\left(x_{p}, L\right]\end{cases}$

where $q_{h}(y)$ and $q_{p}(y)$ represent the user densities for commuters choosing the auto and $\mathrm{P} \& \mathrm{R}$ modes at location $y$, respectively.

It is assumed that no user will choose the $\mathrm{P} \& \mathrm{R}$ mode between the P\&R facility location $x_{p}$ and the CBD, which can be described as follows:

$\begin{cases}q_{p}(x)=0 & \text { if } x \in\left[0, x_{p}\right] \\ q_{p}(x) \geq 0 & \text { if } x \in\left(x_{p}, L\right]\end{cases}$

Then the travel time from location $x$ to the CBD by car can be expressed as follows:

$$
T_{h}(x)=\left\{\begin{aligned}
\int_{0}^{x} t_{h}^{0}\left[1+A\left(\frac{\int_{w}^{L} q_{h}(y) d y}{C_{w}}\right)\right] d w \quad \text { if } x \in\left[0, x_{p}\right] \\
\int_{0}^{x_{p}} t_{h}^{0}\left[1+A\left(\frac{\int_{w}^{L} q_{h}(y) d y}{C_{w}}\right)\right] d w \\
+\int_{x_{p}}^{x} t_{h}^{0}\left[1+A\left(\frac{\int_{w}^{L}\left(q_{h}(y)+q_{p}(y)\right) d y}{C_{w}}\right)\right] d w
\end{aligned}\right.
$$

where $C_{w}$ denotes the stochastic link capacity at location $w$.

Assuming that the link capacity is independent of the traffic volume on it (13), the expectation and variance of travel time on one unit distance at location $x$ can be derived:

$$
\begin{aligned}
E\left(t_{h}\left(v_{h}(x)\right)\right) & =t_{h}^{0}+t_{h}^{0} A v_{h}(x) E\left(C_{x}^{-1}\right) \\
& =t_{h}^{0}+\frac{t_{h}^{0} A v_{h}(x)\left(\ln C_{\max }-\ln C_{\min }\right)}{C_{\max }-C_{\min }} \\
D\left(t_{h}\left(v_{h}(x)\right)\right) & =t_{h}^{0^{2}} A^{2} v_{h}(x)^{2} D\left(C_{x}^{-1}\right)=t_{h}^{0^{2}} A^{2} v_{h}(x)^{2}\left(E\left(C_{x}^{-2}\right)-E\left(C_{x}^{-1}\right)^{2}\right) \\
& =t_{h}^{0^{2}} A^{2} v_{h}(x)^{2}\left[\frac{1}{C_{\max } C_{\min }}-\frac{\left(\ln C_{\max }-\ln C_{\min }\right)^{2}}{\left(C_{\max }-C_{\min }\right)^{2}}\right]
\end{aligned}
$$


To analyze the travel time between any two locations on the corridor, the highway is divided into $n$ unit sections and it is assumed that $T_{h}\left(x_{i}, x_{j}\right)$ is the travel time from section $x_{i}$ to section $x_{j}$; then

$$
\begin{aligned}
& T_{h}\left(x_{i}, x_{j}\right)=\sum_{k=i}^{j} t_{h}\left(v_{h}\left(x_{k}\right)\right) \\
& E\left(T_{h}\left(x_{i}, x_{j}\right)\right)=\sum_{k=i}^{j} E\left(t_{h}\left(v_{h}\left(x_{k}\right)\right)\right) \\
&=\sum_{k=i}^{j}\left[t_{h}^{0}+\frac{t_{h}^{0} A v_{h}(x)\left(\ln C_{\text {max }}-\ln C_{\min }\right)}{C_{\text {max }}-C_{\text {min }}}\right]
\end{aligned}
$$

$D\left(T_{h}\left(x_{i}, x_{j}\right)\right)=\sum_{k=i}^{j} D\left(t_{h}\left(v_{h}\left(x_{k}\right)\right)\right)+2 \sum_{a<b} \operatorname{cov}\left(t_{h}\left(v_{h}\left(x_{a}\right)\right), t_{h}\left(v_{h}\left(x_{b}\right)\right)\right)$,

$$
i \leq a<b \leq j
$$

where $k, i, j, a$, and $b$ are indexes for the road sections.

As shown in Equation 12, when the travel time variance is estimated between location $x_{i}$ and $x_{j}$, there are two items to be calculated; although the first item can be readily obtained from Equation 9, the second item entails the covariance among the travel times traversing each unit distance on the highway between $x_{i}$ and $x_{j}$, which stems from the correlated link capacity among neighboring highway sections. To calculate this item,

$$
\begin{aligned}
\operatorname{cov}\left(t_{h}\left(v_{h}\left(x_{a}\right)\right), t_{h}\left(v_{h}\left(x_{b}\right)\right)\right) & =\operatorname{cov}\left(\begin{array}{c}
t_{h}^{0}+t_{h}^{0} A v_{h}\left(x_{a}\right) C_{x_{a}}^{-1}, \\
t_{h}^{0}+t_{h}^{0} A v_{h}\left(x_{b}\right) C_{x_{b}}^{-1}
\end{array}\right) \\
& =\operatorname{cov}\left(t_{h}^{0} A v_{h}\left(x_{a}\right) C_{x_{a}}^{-1}, t_{h}^{0} A v_{h}\left(x_{b}\right) C_{x_{b}}^{-1}\right) \\
& =t_{h}^{0^{2}} A^{2} v_{h}\left(x_{a}\right) v_{h}\left(x_{b}\right) \operatorname{cov}\left(C_{x_{a}}^{-1}, C_{x_{b}}^{-1}\right) \\
& =t_{h}^{0^{2}} A^{2} v_{h}\left(x_{a}\right) v_{h}\left(x_{b}\right)\left[\begin{array}{l}
E\left(C_{x_{a}}^{-1} C_{x_{b}}^{-1}\right) \\
-E\left(C_{x_{a}}^{-1}\right) E\left(C_{x_{b}}^{-1}\right)
\end{array}\right]
\end{aligned}
$$

The item $E\left(C_{x_{a}}^{-1} C_{x_{b}}^{-1}\right)$ in Equation 13 can be computed if the joint probability distribution function of the stochastic capacities on any two link sections is known. In this study, it is assumed that the correlation, as well as the joint probability distribution of the SLC variables between link sections, can be obtained from the historical data and exogenously given. When the correlation between link capacities of different sections is assumed to be negligible, the item $E\left(C_{x_{a}}^{-1} C_{x_{b}}^{-1}\right)$ in Equation 13 will reduce to zero. This assumption is reasonable for a highway system with relatively few network disruptions. In the following analysis, the assumption of independent SLC is retained for illustrative purposes, whereas the analysis process is still applicable to the situation without this assumption.

\section{Travel Time Budget}

Regardless of the unit travel time distribution, the highway travel time between two locations follows a normal distribution according to the central limit theorem as Lindeberg's condition is satisfied. As was shown by Lo et al., when the travel time for auto users is considered to be stochastic, travelers would reserve some additional time to avoid late arrival, namely, a travel time budget (11). Here, the travel time budget can be expressed as

$T_{b}=E(T)+\lambda \sigma_{T}$

where

$T_{b}=$ travel time budget;

$T=$ travel time on highway;

$E(T), \sigma_{T}=$ expectation and standard deviation of $T$, respectively; and

$\lambda=$ parameter related to punctuality probability requirement $\rho$, which can be mathematically described as follows:

$P\left\{T \leq T_{b}\right\}=P\left\{T \leq E(T)+\lambda \sigma_{T}\right\}=\rho$

Equation 15 is rearranged to obtain the following:

$P\left\{\frac{T-E(T)}{\sigma_{T}} \leq \lambda\right\}=\rho$

Because $[T-E(T)] / \sigma_{T}$ is the standard normal variable, the cumulative distribution function $\Phi(x)$ of the standard normal variable can be written as

$\Phi(\lambda)=\rho$

The inverse is

$\lambda=\Phi^{-1}(\rho)$

Accordingly, larger $\lambda$ leads to a higher punctuality requirement $\rho$, and vice versa. For example, to arrive within a budget time reliability $\rho=95 \%$, the parameter should be set as $\lambda=1.64$. Then the travel time budget expression is

$T_{b}=E(T)+1.64 \sigma_{T}$

Equation 19 implies that travelers have a $95 \%$ probability of arriving on time within the travel time budget.

\section{Generalized Travel Cost}

Commuters choose their travel mode to minimize the generalized travel cost, and user equilibrium status will be achieved with the multimodal choice pattern.

\section{By Auto}

The generalized travel cost by auto is composed of three major components: travel time cost, operating cost, and parking fee. The travel time cost includes two parts: access and egress time and travel time budget $T_{b}(x)$, as was defined in the last section. The operating cost is assumed to be relevant to the travel distance. 
The generalized travel cost by auto from location $x$ to the CBD is

$C_{h}(x)=\tau t_{h}^{h h}+\tau t_{h}^{p w}+\tau T_{b}(x)+f_{h}(x)+P_{h}^{w}$

where

$\tau=$ value of time,

$t_{h}^{h h}=$ access time from home to highway, and

$t_{h}^{p w}=$ access time from parking place to workplace.

The operating cost is assumed to be a linear function of the travel distance, $f_{h}(x)=f_{h}^{0}+\gamma x$, where $f_{h}^{0}$ is a constant that may include a fixed part of the tolls, and $\gamma x$ presents the variable part of the operating cost from location $x$ to the CBD, such as the fuel, insurance, and variable parts of the highway tolls, and so forth, with $\gamma$ as the operating cost per unit distance of travel. $P_{h}^{w}$ is the parking fee at the CBD.

\section{By Railway}

As with auto users, the traffic volume of transit users at location $x$ by railway is calculated as

$v_{r}(x)= \begin{cases}\int_{x}^{L}\left(q_{r}(w)+q_{p}(w)\right) d w & \text { if } x \in\left[0, x_{p}\right] \\ \int_{x}^{L} q_{r}(w) d w, & \text { if } x \in\left(x_{p}, L\right]\end{cases}$

where $q_{r}(w)$ is the user density for commuters choosing the public transit mode at location $w$.

The crowding cost is considered here to reflect the loss of privacy and comfort, decline of air quality, and advent of pickpockets due to the increase in passengers on the train. Let $g_{r}\left(v_{r}(x)\right)$ be the crowding cost on the train per unit distance at location $x$, where $v_{r}(x)$ represents the number of passengers by railway at location $x$. As was done by Huang, a simple linear function with respect to traffic volume on the train is applied to describe the crowding cost (15):

$g_{r}\left(v_{r}(x)\right)=\alpha+\beta v_{r}(x)$

where $\alpha, \beta$ are the calibrated parameters. Therefore, the crowding cost for commuters traveling from location $x$ to the CBD should be

$G_{r}(x)=\int_{0}^{x} g_{r}\left(v_{r}(w)\right) d w$

The crowding cost can be calculated as follows:

$G_{r}(x)=\left\{\begin{aligned} \int_{0}^{x}\left[\alpha+\beta \int_{w}^{L}\left(q_{r}(y)+q_{p}(y)\right) d y\right] d w & \text { if } x \in\left[0, x_{p}\right] \\ \int_{0}^{x_{p}}\left[\alpha+\beta \int_{w}^{L}\left(q_{r}(y)+q_{p}(y)\right) d y\right] d w & \\ \quad+\int_{x_{p}}^{x}\left(\alpha+\beta \int_{w}^{L} q_{r}(y) d y\right) d w & \text { if } x \in\left(x_{p}, L\right]\end{aligned}\right.$

The generalized travel cost by rail transit from location $x$ to the CBD is composed of three major components: travel time cost, crowding cost, and service fare:

$C_{r}(x)=\tau t_{r}^{h r}+\tau t_{r}^{m w}+\tau \frac{x}{V_{r}}+G_{r}(x)+f_{r}(x)$ where

$t_{r}^{h r}, t_{r}^{r w}=$ access and egress time, respectively;

$x / V_{r}=$ travel time by railway from location $x$ to CBD where $V_{r}$ is the average travel speed of the train; and

$f_{r}(x)=f_{r}^{0}+\kappa x$

$=$ fare structure of railway service

where

$f_{r}^{0}=$ fixed part of the fare,

$\kappa x=$ variable component service fare from location $x$ to CBD, and

$\kappa=$ railway fare per unit distance.

\section{By P\&R}

The generalized travel cost by $\mathrm{P} \& \mathrm{R}$ has three major parts: the auto mode cost, the $P \& R$ transfer cost, and the transit mode cost; it is assumed that no one will choose the $\mathrm{P} \& \mathrm{R}$ mode from $\mathrm{P} \& \mathrm{R}$ facility location $x_{p}$ to CBD.

$C_{p}(x)=\left\{\begin{array}{rr}\boldsymbol{U} & \text { if } x \in\left[0, x_{p}\right] \\ \tau t_{h}^{h h}+\tau\left(T_{b}(x)-T_{b}\left(x_{p}\right)\right)+f_{h}\left(x, x_{p}\right)+p_{p}\left(x_{p}\right) \\ +\tau t_{p}^{p r}+u_{p}+\tau \frac{x_{p}}{V_{r}}+G_{r}\left(x_{p}\right)+f_{r}\left(x_{p}\right)+\tau t_{r}^{r w} \\ & \text { if } x \in\left(x_{p}, L\right]\end{array}\right.$

where $\boldsymbol{U}$ represents a large enough positive constant to describe the generalized travel cost because no one will choose P\&R from location $x_{p}$ to CBD.

At location $x \in\left(x_{p}, L\right]$, the generalized travel cost can be divided into three parts:

Auto mode cost:

$\tau t_{h}^{h h}+\tau\left(T_{b}(x)-T_{b}\left(x_{p}\right)\right)+f_{h}\left(x, x_{p}\right)$

where $T_{b}(x)-T_{b}\left(x_{p}\right)$ and $f_{h}\left(x, x_{p}\right)$ are the travel time budget and operating cost from location $x$ to P\&R facility $x_{p}$.

$P \& R$ transfer cost:

$p_{p}\left(x_{p}\right)+\tau t_{p}^{p r}+u_{p}$

where $p_{p}\left(x_{p}\right)$ represents the parking fee at $\mathrm{P} \& \mathrm{R}$ facility location $x_{p}$, which, according to Liu et al., (10) can be calculated as

$p_{p}\left(x_{p}\right)=p_{h}^{w} e^{-\frac{x_{p}^{2}}{2 L}}$

where $t_{p}^{p r}$ is the transfer time from parking place to nearby train station, and $u_{p}$ is the inconvenience or disutility cost.

Transit mode cost:

$\tau \frac{x_{p}}{V}+G_{r}\left(x_{p}\right)+f_{r}\left(x_{p}\right)+\tau t_{r}^{r w}$

where $x_{p} / V$ is the travel time from P\&R location $x_{p}$ to CBD workplace, $G_{r}\left(x_{p}\right)$ is the crowding cost from location $x_{p}$ to CBD, and $f_{r}\left(x_{p}\right)$ is the service fare from location $x_{p}$ to CBD. 


\section{SPATIAL EQUILIBRIUM WITH MULTIMODAL CHOICE}

User equilibrium is achieved when no travelers can reduce their generalized travel cost by using other modes at any location. This spatial user equilibrium is modeled by applying the complementarity system approach as follows:

$$
\begin{aligned}
& 0 \leq q_{h}(x) \perp C_{h}(x)-C^{*}(x) \geq 0 \\
& 0 \leq q_{r}(x) \perp C_{r}(x)-C^{*}(x) \geq 0 \\
& 0 \leq q_{p}(x) \perp C_{p}(x)-C^{*}(x) \geq 0 \\
& 0 \leq C^{*}(x) \perp q_{h}(x)+q_{r}(x)+q_{p}(x)-q_{0}(x) \geq 0 \\
& x \in[0, L]
\end{aligned}
$$

where $C^{*}(x)$ represents the minimum generalized travel cost among the three modes at location $x$. The first three constraints in Equation 27 ensure that at equilibrium, the individual travel cost by a mode at any location is the minimum among the three modes if the mode is used at the location. The last complementarity constraint ensures that total users by the three modes amount to the given total travel demand density $q_{0}(x)$ at location $x$.

\section{LINEAR COMPLEMENTARITY SYSTEM FORMULATION}

To formulate the spatial equilibrium travel pattern with multimodal choice into an LCS, first, if the second-order derivative of the travel time budget $T_{b}(x)$ with respect to $x$ is taken,

$$
\frac{d^{2} T_{b}(x)}{d x^{2}}= \begin{cases}-\boldsymbol{D} \cdot q_{h}(x) & \text { if } x \in\left[0, x_{p}\right) \\ -\boldsymbol{D} \cdot\left(q_{h}(x)-\int_{x_{p}}^{L} q_{p}(y) d y\right) & \text { if } x=x_{p} \\ -\boldsymbol{D} \cdot\left(q_{h}(x)+q_{p}(x)\right) & \text { if } x \in\left(x_{p}, L\right]\end{cases}
$$

where $\boldsymbol{D}$ denotes the constant part in Equation 28 as follows:

$$
\begin{aligned}
\boldsymbol{D}= & {\left[\frac{t_{h}^{0} A\left(\ln C_{\max }-\ln C_{\min }\right)}{C_{\max }-C_{\min }}\right.} \\
& \left.+\lambda t_{h}^{0} A \sqrt{\frac{1}{C_{\max } C_{\min }}-\left(\frac{\ln C_{\max }-\ln C_{\min }}{C_{\max }-C_{\min }}\right)^{2}}\right]
\end{aligned}
$$

Similarly, the second-order derivative of the crowding cost of the transit mode is obtained:

$$
\frac{d^{2} G_{r}(x)}{d x^{2}}= \begin{cases}-\beta \cdot\left(q_{r}(x)+q_{p}(x)\right) & \text { if } x \in\left[0, x_{p}\right) \\ -\beta \cdot\left(q_{r}(x)+\int_{x_{p}}^{L} q_{p}(y) d y\right) & \text { if } x=x_{p} \\ -\beta \cdot q_{r}(x) & \text { if } x \in\left(x_{p}, L\right]\end{cases}
$$

The previous ordinary differential equations (ODEs) reflect the spatial interaction of the travel time and crowding cost along the corridor. Furthermore, initial conditions and boundary conditions of the ODEs can be expressed as follows:

$T_{b}(0)=0, G_{r}(0)=0$ $\frac{d T_{b}}{d x}(x=L)=t_{h}^{0} \quad \frac{d G_{r}}{d x}(x=L)=\alpha$

The initial conditions ensure that the travel time and crowding cost at the city center will be zero. The boundary conditions entail that no travel demand will be generated beyond the corridor length $L$; that is, $v_{h}(L)=v_{r}(L)=0$.

From the foregoing analysis, an LCS can be used to describe the spatial equilibrium travel pattern with multimodal choice. The solution is to find $T_{b}(x), G_{r}(x), q_{h}(x), q_{r}(x), q_{p}(x)$, and $C^{*}(x)$ so that following conditions are satisfied:

1. For almost all $x \in(0, L]$ :

$\frac{d^{2} T_{b}(x)}{d x^{2}}= \begin{cases}-\boldsymbol{D} \cdot q_{h}(x) & \text { if } x \in\left[0, x_{p}\right) \\ -\boldsymbol{D} \cdot\left(q_{h}(x)-\int_{x_{p}}^{L} q_{p}(y) d y\right) & \text { if } x=x_{p} \\ -\boldsymbol{D} \cdot\left(q_{h}(x)+q_{p}(x)\right) & \text { if } x \in\left(x_{p}, L\right]\end{cases}$
$\frac{d^{2} G_{r}(x)}{d x^{2}}= \begin{cases}-\beta \cdot\left(q_{r}(x)+q_{p}(x)\right) & \text { if } x \in\left[0, x_{p}\right) \\ -\beta \cdot\left(q_{r}(x)+\int_{x_{p}}^{L} q_{p}(y) d y\right) & \text { if } x=x_{p} \\ -\beta \cdot q_{r}(x) & \text { if } x \in\left(x_{p}, L\right]\end{cases}$

$0 \leq q_{h}(x) \perp C_{h}(x)-C^{*}(x) \geq 0$

$0 \leq q_{r}(x) \perp C_{r}(x)-C^{*}(x) \geq 0$

$0 \leq q_{p}(x) \perp C_{p}(x)-C^{*}(x) \geq 0$

$0 \leq C^{*}(x) \perp q_{h}(x)+q_{r}(x)+q_{p}(x)-q_{0}(x) \geq 0$

$x \in[0, L]$

2. For the initial and boundary conditions:

$T_{b}(0)=0, \quad G_{r}(0)=0$
$\frac{d T_{b}}{d x}(x=L)=t_{h}^{0}, \quad \frac{d G_{r}}{d x}(x=L)=\alpha$

For illustrative purposes, $C_{h}(x), C_{r}(x)$, and $C_{p}(x)$ are used in Equation 33 to represent the generalized travel costs by auto, railway, and P\&R modes, respectively, and the complete formulations are shown in the previous Equations 20, 25, and 26, respectively.

\section{DISCRETE APPROXIMATION OF LCS MODEL}

The numerical solution of the formulated LCS (Equation 33) can be accomplished via a time-stepping scheme that solves a sequence of finite-dimensional discretized subproblems. Specifically, the corridor length is divided into equal-length subintervals $\varepsilon_{v}=L / v$ by a positive integer $v>0$. Then the numerical solution of the LCS is obtained by computing the discretized scheme:

$\left\{q_{h}^{i}\right\},\left\{q_{r}^{i}\right\},\left\{q_{p}^{i}\right\},\left\{C^{*, i}\right\},\left\{T_{b}^{i}\right\},\left\{G_{r}^{i}\right\} \quad i=1,2, \ldots, v$

In the discretization scheme, the derivative can be approximated by the forward difference quotient:

$\frac{d^{2} T_{b}(x)}{d x^{2}} \approx \frac{\left[T_{b}\left(x+\varepsilon_{v}\right)-T_{b}(x)\right]-\left[T_{b}(x)-T_{b}\left(x-\varepsilon_{v}\right)\right]}{\varepsilon_{v}^{2}}$ 
$\frac{d^{2} G_{r}(x)}{d x^{2}} \approx \frac{\left[G_{r}\left(x+\varepsilon_{v}\right)-G_{r}(x)\right]-\left[G_{r}(x)-G_{r}\left(x-\varepsilon_{v}\right)\right]}{\varepsilon_{v}^{2}}$

Therefore, the discretized approximation of Condition 1 is

$\frac{T_{b}^{i+1}+T_{b}^{i-1}-2 T_{b}^{i}}{\varepsilon_{v}^{2}}= \begin{cases}-\boldsymbol{D} \cdot q_{h}^{i} & \text { if } 1 \leq i<i^{x_{p}} \\ -\boldsymbol{D} \cdot\left(q_{h}^{i}-\sum_{j=i^{x_{p}}+1}^{v} q_{p}^{j}\right) & \text { if } i=i^{x_{p}} \\ -\boldsymbol{D} \cdot\left(q_{h}^{i}+q_{p}^{i}\right) & \text { if } i^{x_{p}}<i \leq v\end{cases}$
$\frac{G_{r}^{i+1}+G_{r}^{i-1}-2 G_{r}^{i}}{\varepsilon_{v}^{2}}= \begin{cases}-\beta \cdot\left(q_{r}^{i}+q_{p}^{i}\right) & \text { if } 1 \leq i<i^{x_{p}} \\ -\beta \cdot\left(q_{r}^{i}+\sum_{j=i^{x_{p}}}^{v} q_{p}^{j}\right) & \text { if } i=i^{x_{p}} \\ -\beta \cdot q_{r}^{i} & \text { if } i^{x_{p}}<i \leq v\end{cases}$

$0 \leq q_{h}^{i} \perp\left(\tau t_{h}^{h h}+\tau t_{h}^{p w}+\tau T_{b}^{i}+f_{h}^{0}+\gamma i \varepsilon_{v}+P_{h}^{w}-C^{*, i}\right) \geq 0$

$0 \leq q_{r}^{i} \perp\left(\tau \tau_{r}^{h r}+\tau t_{r}^{h w}+\tau \frac{i \varepsilon_{v}}{V_{r}}+G_{r}^{i}+f_{r}^{0}+\kappa i \varepsilon_{v}-C^{*, i}\right) \geq 0$

$0 \leq q_{p}^{i} \perp\left\{\begin{array}{ll}\left(\boldsymbol{U}-C^{* i}\right) \geq 0 & \text { if } 1 \leq i \leq i^{x_{p}} \\ \left(\tau t_{h}^{h h}+\tau\left(T_{b}^{i}-T_{b}^{x_{p}^{x_{p}}}\right)+f_{h}^{0}\right. \\ +\gamma\left(i \varepsilon_{v}-i^{x_{p}} \varepsilon_{v}\right)+p_{h}^{w} e^{-\frac{x_{p}^{2}}{2 L}} \\ +\tau t_{p}^{p r}+u_{p}+\tau \frac{i^{x_{p}} \varepsilon_{v}}{V_{r}}+G_{r}^{t_{p}^{x_{p}}} \\ +f_{r}^{0}+\kappa i^{x_{p}} \varepsilon_{v}+\tau t_{r}^{w_{w}}-C^{*, i}\end{array}\right) \geq 0 \quad$ if $i^{x_{p}}<i \leq v$

$0 \leq C^{*, i} \perp q_{h}^{i}+q_{r}^{i}+q_{p}^{i}-q_{0}^{i} \geq 0$

$i=1,2, \ldots, v$

where $i^{x_{p}}$ represents the section where P\&R facility is located.

Let

$\eta= \begin{cases}0 & \text { if } 1 \leq i<i^{x_{p}} \\ 1 & \text { if } i^{x_{p}}<i \leq v\end{cases}$

Where $\eta$ represents a binary variable. Then

$\frac{T_{b}^{i+1}+T_{b}^{i-1}-2 T_{b}^{i}}{\varepsilon_{v}^{2}}= \begin{cases}-\boldsymbol{D} \cdot\left(q_{h}^{i}+\eta \cdot q_{p}^{i}\right) & \text { if } i \neq i^{x_{p}} \\ -\boldsymbol{D} \cdot\left(q_{h}^{i}-\sum_{j=i^{x_{p}^{p}}+1}^{v} q_{p}^{j}\right) & \text { if } i=i^{x_{p}}\end{cases}$

$\frac{G_{r}^{i+1}+G_{r}^{i-1}-2 G_{r}^{i}}{\varepsilon_{v}^{2}}= \begin{cases}-\beta \cdot\left[q_{r}^{i}+(1-\eta) \cdot q_{p}^{i}\right] & \text { if } i \neq i^{x_{p}} \\ -\beta \cdot\left(q_{r}^{i}+\sum_{j=i^{x_{p}}}^{v} q_{p}^{j}\right) & \text { if } i=i^{x_{p}}\end{cases}$

Because $T_{b}^{i}>0, G_{r}^{i}>0, i=1,2, \ldots, v$ can convert the first two equations into a standard form of linear complementarity problem (LCP), the following equivalent LCP can be obtained in which the variables are $\left(q_{h}^{i}\right)_{i=1}^{v},\left(q_{r}^{i}\right)_{i=1}^{v},\left(q_{p}^{i}\right)_{i=1}^{v},\left(C^{*, i}\right)_{i=1}^{v},\left(T_{b}^{i}\right)_{i=1}^{v},\left(G_{r}^{i}\right)_{i=1}^{v}, i=1$, $2, \ldots, v$.
$0 \leq T_{b}^{i} \perp \begin{cases}T_{b}^{i+1}+T_{b}^{i-1}-2 T_{b}^{i}+\varepsilon_{v}^{2} \boldsymbol{D} \cdot\left(q_{h}^{i}+\eta \cdot q_{p}^{i}\right) \geq 0 & \text { if } i \neq i^{x_{p}} \\ T_{b}^{i+1}+T_{b}^{i-1}-2 T_{b}^{i}+\varepsilon_{v}^{2} \boldsymbol{D} \cdot\left(q_{h}^{i}-\sum_{j=i^{x_{p}}}^{v} q_{p}^{j}\right) \geq 0 & \text { if } i=i^{x_{p}}\end{cases}$ $0 \leq G_{r}^{i} \perp \begin{cases}G_{r}^{i+1}+G_{r}^{i-1}-2 G_{r}^{i}+\varepsilon_{v}^{2} \beta \cdot\left[q_{r}^{i}+(1-\eta) \cdot q_{p}^{i}\right] \geq 0 & \text { if } i \neq i^{x_{p}} \\ G_{r}^{i+1}+G_{r}^{i-1}-2 G_{r}^{i}+\varepsilon_{v}^{2} \beta \cdot\left[q_{r}^{i}+\sum_{j=i^{x_{p}}}^{v} q_{p}^{j}\right] \geq 0 & \text { if } i=i^{x_{p}}\end{cases}$ $0 \leq q_{h}^{i} \perp\left(\tau t_{h}^{h h}+\tau t_{h}^{p w}+\tau T_{b}^{i}+f_{h}^{0}+\gamma i \varepsilon_{v}+P_{h}^{w}-C^{*, i}\right) \geq 0$

$0 \leq q_{r}^{i} \perp\left(\tau t_{r}^{h r}+\tau t_{r}^{n w}+\tau \frac{i \varepsilon_{v}}{V_{r}}+G_{r}^{i}+f_{r}^{0}+\kappa i \varepsilon_{v}-C^{*, i}\right) \geq 0$

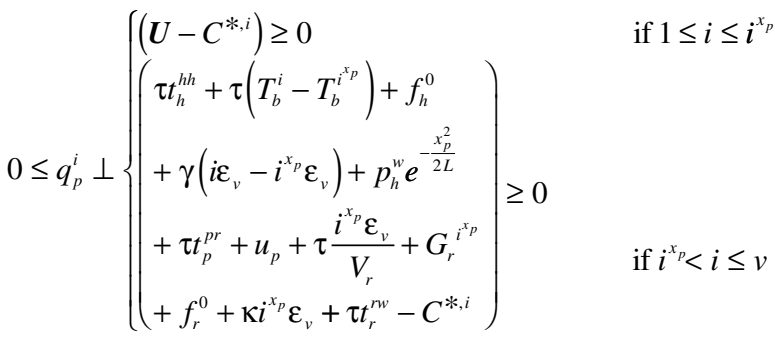

$0 \leq C^{*, i} \perp q_{h}^{i}+q_{r}^{i}+q_{p}^{i}-q_{0}^{i} \geq 0$

$i=1,2, \ldots, v$

The initial and boundary Conditions 2 can be rewritten as

$T_{b}^{0}=0, G_{r}^{0}=0$

$T_{b}^{v+1}-T_{b}^{v}=t_{h}^{0} \varepsilon_{v}, G_{r}^{v+1}-G_{r}^{v}=\alpha \varepsilon_{v}$

Equation 42 can be substituted into Equation 41 directly to simplify the formulation.

The LCP model in a standard form can be expressed as follows to find a vector $x$ satisfying

$0 \leq x \perp M x+q \geq 0$

where

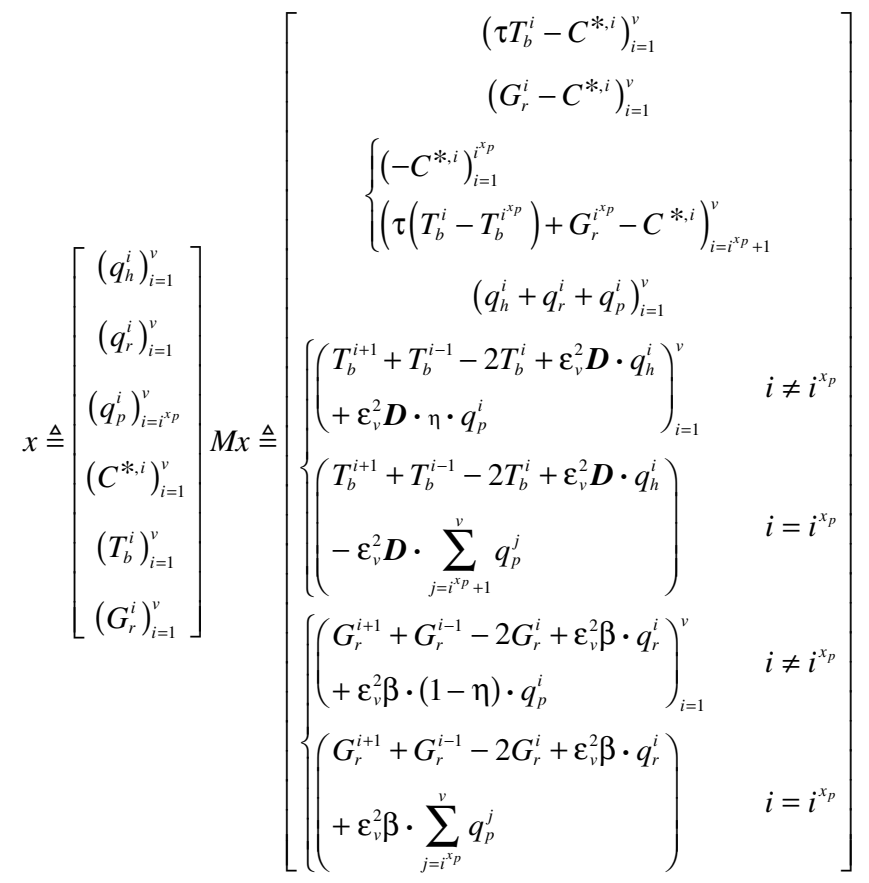




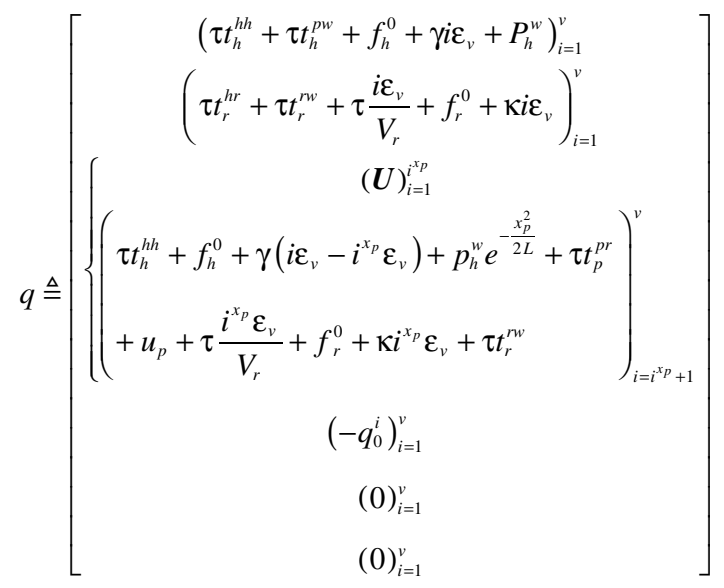

\section{TRANSFORMATION OF LCP TO MIXED INTEGER LINEAR PROGRAM}

In this study, an equivalent mixed integer linear program (MILP) method is used to reformulate the LCP. First, a bilinear formulation approach is used to state the LCP as a mixed integer bilinear program (MIBLP) as follows:

$\min y^{T}(M x+q)+(e-y)^{T} x$

subject to

$x \geq 0$

$M x+q \geq 0$

$y$ binary

where $e$ presents the vector of $n$ ones.

Proposition 1. The solution of the proposed LCP (Equation 41) is equivalent to the solution of the MIBLP problem (Equation 45), with zero as the minimal objective value.

Proof. If $x$ solves the original LCP, define

$y_{i}= \begin{cases}0 & \text { if } M_{i} x+q_{i}>0 \\ 1 & \text { if } x_{i}>0\end{cases}$

Then the $x$ solution of the problem in Equation 41 renders zero as the objective value of the MIBLP problem in Equation 45. If $x$ solves Equation 45 with zero as the minimal objective value, $y^{T}(M x+q)+(e-y)^{T} x=0$. Because $y$ is binary, either $y^{T}$ or $(e-y)^{T}$ must be zero and another one must be 1 . In that case, either $M x+q$ or $x$ must be zero so that $y^{T}(M x+q)+(e-y)^{T} x=0$; then $x^{T}(M x+$ $q)=0$ is also fulfilled, which is the solution to the LCP problem in Equation 41 as well.

Let $M_{i j}$ denote the $(i, j)$ th element of $M$; Equation 46 can be rewritten as follows:

$\min q^{T} y+\sum_{i=1}^{n} \sum_{j=1}^{n} M_{i j} y_{i} x_{j}+(e-y)^{T} x$ subject to

$\sum_{j=1}^{n} M_{k j} x_{j}+q_{k} \geq 0 \quad k=1,2, \ldots, n$

$x \geq 0, y$ binary

Finally, the reformulation-linearization technique is applied to convert the MIBLP into an MILP problem (16). This method consists of two phases: the reformation phase and the linearization phase. The reformation phase defines a set of nonnegative variable factors and then forms the products of these factors with original constraints to generate various implied nonlinear restrictions. In the linearization phase, a variable substitution technique is used to linearize these nonlinear constraints. This method transforms the problem into a higher-dimensional space problem such that its continuous relaxation approximates the closure of the convex hull of feasible solutions to the underlying mixed integer program problem. Specifically, to reformulate the problem, the constraints in Equation 45 are multiplied by $\left(1-y_{i}\right)$ and $y_{i}$ and then the problem is linearized by substituting $w_{i j}=y_{i} x_{j}$. The equivalent MILP is as follows:

$\min q^{T} y+\sum_{i=1}^{n} \sum_{j=1}^{n} M_{i j} w_{i j}$

subject to

$\sum_{j=1}^{n} M_{k j} w_{i j}+q_{k} y_{i} \geq 0 \quad \forall(i, k)$

$\sum_{j=1}^{n} M_{k j} x_{j}+q_{k} \geq \sum_{j=1}^{n} M_{k j} w_{i j}+q_{k} y_{i} \quad \forall(i, k)$

$0 \leq w_{i j} \leq x_{j}, \forall(i, j) \quad$ with $w_{j j}=x_{j}, \forall j$

$x \geq 0, \quad y$ binary

For the proof of the equivalence of the MILP in Equation 48 with the MIBLP in Equation 45, one can refer to the work by Sherali et al. (16). To solve the MILP in Equation 48, commercial software such as CPLEX can be used to obtain the final solution.

\section{NUMERICAL RESULTS}

In this section, a set of numerical results is presented for an example corridor. The corridor is divided into $v$ sections, and the deterministic use equilibrium mode choice is achieved on each section. On each section the link capacity is stochastic and follows a uniform distribution.

The input data for the parameters are shown in Table 1, and the corridor is divided into 20 sections. Sections from 1 to 20 represent the locations from the CBD to the boundary of the corridor, and the P\&R facility location is supposed to be at Section 10 $\left(x_{p}=10\right)$. It is assumed that $\mathrm{P} \& \mathrm{R}$ is not available from the $\mathrm{P} \& \mathrm{R}$ facility location to the CBD, so only commuters living between the $P \& R$ facility and the corridor boundary can choose P\&R. First, the mode split pattern at equilibrium for the scenario is examined considering travel time reliability with probability requirement $\rho=95 \%$.

The mode split pattern at equilibrium with SLC is given in Table 2. All three modes are used along the corridor, and the rail- 
TABLE 1 Parameter Values and Descriptions

\begin{tabular}{|c|c|c|}
\hline Parameter & Value & Description \\
\hline$L$ & 20 & Length of corridor (km) \\
\hline$v$ & 20 & Section number of corridor \\
\hline$x_{p}$ & 10 & Location of P\&R facility \\
\hline$q_{0}$ & 800 & Commuter density (commuters/h/km) \\
\hline$\tau$ & 0.5 & Time-to-cost coefficient ( $\$ \$ / \min )$ \\
\hline$\rho$ & $95 \%$ & $\begin{array}{l}\text { Probability that trip arrives within travel } \\
\text { time budget }\end{array}$ \\
\hline$\lambda$ & 1.64 & Parameter related to $\rho$ \\
\hline$A$ & 0.5 & BPR function parameter \\
\hline$t_{h}^{0}$ & 1 & $\begin{array}{l}\text { BPR function parameter, free-flow travel } \\
\text { time (min) }\end{array}$ \\
\hline$C_{\max }$ & 15,000 & Maximal link capacity (veh/km) \\
\hline$C_{\min }$ & 5,000 & Minimal link capacity (veh/km) \\
\hline$C$ & 10,000 & $\begin{array}{l}\text { Link capacity without considering } \\
\text { reliability }(\mathrm{veh} / \mathrm{km})\end{array}$ \\
\hline$t_{h}^{h h}$ & 2 & Time from home to highway (min) \\
\hline$t_{h}^{p w}$ & 2 & $\begin{array}{l}\text { Time from parking place to workplace } \\
\text { (min) }\end{array}$ \\
\hline$f_{h}^{0}$ & 2 & $\begin{array}{l}\text { Fixed part of the operating cost by auto } \\
\text { (S\$/veh) }\end{array}$ \\
\hline$\gamma$ & 0.03 & $\begin{array}{l}\text { Operating cost per unit distance by auto } \\
(\mathrm{S} \$ / \mathrm{km} / \mathrm{veh})\end{array}$ \\
\hline$p_{h}^{w}$ & 8 & Parking fee at workplace (S\$/veh) \\
\hline$t_{r}^{h r}$ & 12 & Time from home to railway station ( $\mathrm{min})$ \\
\hline$t_{r}^{r w}$ & 5 & $\begin{array}{l}\text { Time from railway station to workplace } \\
\text { (min) }\end{array}$ \\
\hline$f_{r}^{0}$ & 0.5 & Fixed part of fare ( $\mathrm{S} \$$ ) \\
\hline$\kappa$ & 0.06 & Railway fare per unit distance (S\$/km) \\
\hline$V_{r}$ & 0.8 & Average speed of train (km/min) \\
\hline$\alpha$ & 0.004 & Parameter of crowding cost \\
\hline$\beta$ & 0.000024 & Parameter of crowding cost \\
\hline$t_{p}^{p r}$ & 1 & $\begin{array}{l}\text { Time from parking place at } P \& R \text { location } \\
\text { to train station nearby (min) }\end{array}$ \\
\hline$u_{p}$ & 1 & Fixed transfer disutility \\
\hline
\end{tabular}

NOTE: BPR = Bureau of Public Roads; $\$$ \$ = Singapore dollars.

way has tremendous advantages at places close to the CBD. In contrast, $\mathrm{P} \& \mathrm{R}$ is preferred from the middle of the corridor to the boundary. Specifically, all users choose railway from Locations 1 to 8 , auto is preferred in the middle part of the corridor at Locations 9 and 10, and $\mathrm{P} \& \mathrm{R}$ is the mode choice from the corridor boundary to Location 11; thus all commuters living between Locations 11 and 20 will drive to Location 10 to transfer to the railway to complete the rest of the trip.

Figure 2 shows the generalized costs of the three modes along the corridor. The minimum cost curve is a combination of three parts. From the CBD to the boundary of the corridor, the generalized cost of the railway is less than that of the other modes from Location 1 to Location 8, and then the lowest generalized cost is by the auto mode at Locations 9 and 10. From Location 11 to the corridor boundary, $\mathrm{P} \& \mathrm{R}$ is preferred according to its lowest generalized cost. The generalized cost of $P \& R$ from Location 1 to 10 is not available since no one will choose $P \& R$ from the $P \& R$ facility location (Section 10) to the $\mathrm{CBD}$, as assumed in the previous discussion.

As is shown in Table 3, the number of $P \& R$ commuters remains unchanged from Location 11 to the corridor boundary; however,
TABLE 2 Mode Split Pattern at Equilibrium Status with SLC

\begin{tabular}{lccc}
\hline Section & $\begin{array}{c}\text { Auto User } \\
\text { Density }\end{array}$ & $\begin{array}{l}\text { Railway User } \\
\text { Density }\end{array}$ & $\begin{array}{c}\text { P\&R User } \\
\text { Density }\end{array}$ \\
\hline 1 & 0 & 800 & 0 \\
2 & 0 & 800 & 0 \\
3 & 0 & 800 & 0 \\
4 & 0 & 800 & 0 \\
5 & 0 & 800 & 0 \\
6 & 0 & 800 & 0 \\
7 & 0 & 800 & 0 \\
8 & 0 & 800 & 0 \\
9 & 800 & 0 & 0 \\
10 & 800 & 0 & 0 \\
11 & 0 & 0 & 800 \\
12 & 0 & 0 & 800 \\
13 & 0 & 0 & 800 \\
14 & 0 & 0 & 800 \\
15 & 0 & 0 & 800 \\
16 & 0 & 0 & 800 \\
17 & 0 & 0 & 800 \\
18 & 0 & 0 & 800 \\
19 & 0 & 0 & 800 \\
20 & 0 & 0 & 800 \\
\hline
\end{tabular}

NOTE: Values are in commuters per hour per kilometer.

some auto users change to the railway at Location 8 if SLC is considered since the travel time budget needs to be considered on the highway, which leads to more generalized cost and fewer users by auto.

Figure 3 illustrates the different generalized costs before and after SLC is considered. All the generalized costs of the three modes increase after SLC is considered, because commuters reserve a large travel time budget for higher reliability; the mode split pattern also changes, wherein more people choose railway and $P \& R$ to take advantage of the higher travel time reliability of rail transit service.

From Figure 4 it can be seen that both travel time on the highway and crowding cost on the railway increase since auto users need to consider the travel time budget. Therefore more commuters use railway or $\mathrm{P} \& \mathrm{R}$ instead of the auto mode, which brings more people to the railway mode and a higher crowding cost.

It is easy to understand that location of the P\&R facility at different places will lead to different multimodal choice patterns. By varying the $P \& R$ location, the optimal location that minimizes the total system travel cost can be found. From Table 4, it can be seen that the best location for the P\&R facility is Location 13, where the minimum total generalized cost is achieved.

\section{CONCLUSIONS}

A reliability-based model of the $\mathrm{P} \& \mathrm{R}$ service problem is considered. Travel time reliability is explicitly incorporated into the mode choice modeling. The spatial equilibrium travel pattern with multimodal choice is formulated into an LCS, which belongs to the class of the DVI modeling paradigm. Then it is transformed into an equivalent MILP by solution of a discrete approximation method. A numerical example is conducted to compare the equilibrium patterns before and 


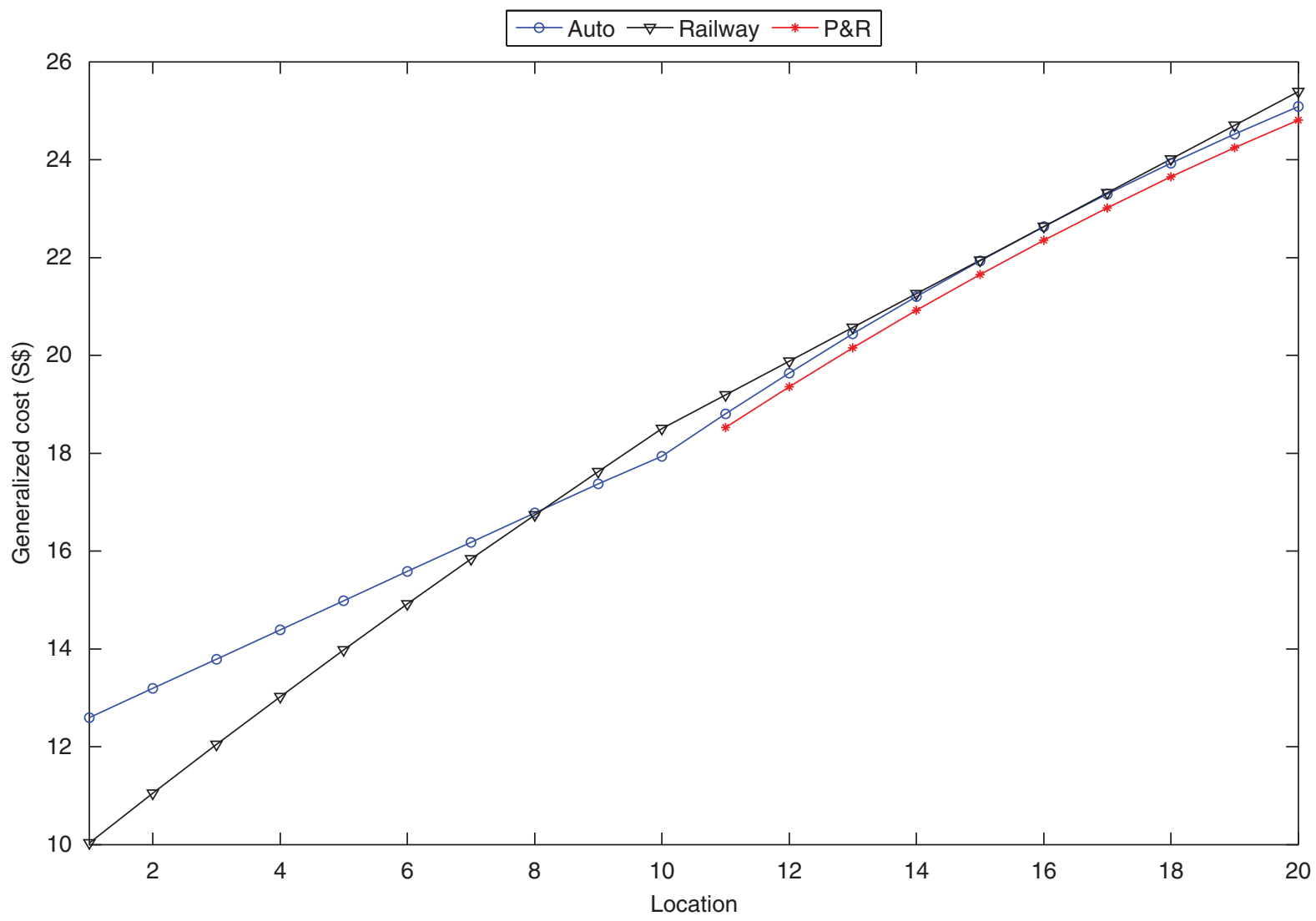

FIGURE 2 Generalized costs of three modes at equilibrium status with SLC.

TABLE 3 Comparison of Mode Split Patterns Between DLC and SLC

\begin{tabular}{|c|c|c|c|c|c|c|}
\hline \multirow[b]{2}{*}{ Section } & \multicolumn{3}{|l|}{ DLC } & \multicolumn{3}{|l|}{ SLC } \\
\hline & $\begin{array}{l}\text { Auto User } \\
\text { Density }\end{array}$ & $\begin{array}{l}\text { Railway User } \\
\text { Density }\end{array}$ & $\begin{array}{l}\text { P\&R User } \\
\text { Density }\end{array}$ & $\begin{array}{l}\text { Auto User } \\
\text { Density }\end{array}$ & $\begin{array}{l}\text { Railway User } \\
\text { Density }\end{array}$ & $\begin{array}{l}\text { P\&R User } \\
\text { Density }\end{array}$ \\
\hline 1 & 0 & 800 & 0 & 0 & 800 & 0 \\
\hline 2 & 0 & 800 & 0 & 0 & 800 & 0 \\
\hline 3 & 0 & 800 & 0 & 0 & 800 & 0 \\
\hline 4 & 0 & 800 & 0 & 0 & 800 & 0 \\
\hline 5 & 0 & 800 & 0 & 0 & 800 & 0 \\
\hline 6 & 0 & 800 & 0 & 0 & 800 & 0 \\
\hline 7 & 0 & 800 & 0 & 0 & 800 & 0 \\
\hline 8 & 457.1429 & 342.8571 & 0 & 0 & 800 & 0 \\
\hline 9 & 800 & 0 & 0 & 800 & 0 & 0 \\
\hline 10 & 800 & 0 & 0 & 800 & 0 & 0 \\
\hline 11 & 0 & 0 & 800 & 0 & 0 & 800 \\
\hline 12 & 0 & 0 & 800 & 0 & 0 & 800 \\
\hline 13 & 0 & 0 & 800 & 0 & 0 & 800 \\
\hline 14 & 0 & 0 & 800 & 0 & 0 & 800 \\
\hline 15 & 0 & 0 & 800 & 0 & 0 & 800 \\
\hline 16 & 0 & 0 & 800 & 0 & 0 & 800 \\
\hline 17 & 0 & 0 & 800 & 0 & 0 & 800 \\
\hline 18 & 0 & 0 & 800 & 0 & 0 & 800 \\
\hline 19 & 0 & 0 & 800 & 0 & 0 & 800 \\
\hline 20 & 0 & 0 & 800 & 0 & 0 & 800 \\
\hline
\end{tabular}

Note: Values are in commuters per hour per kilometer; DLC $=$ deterministic link capacity. 


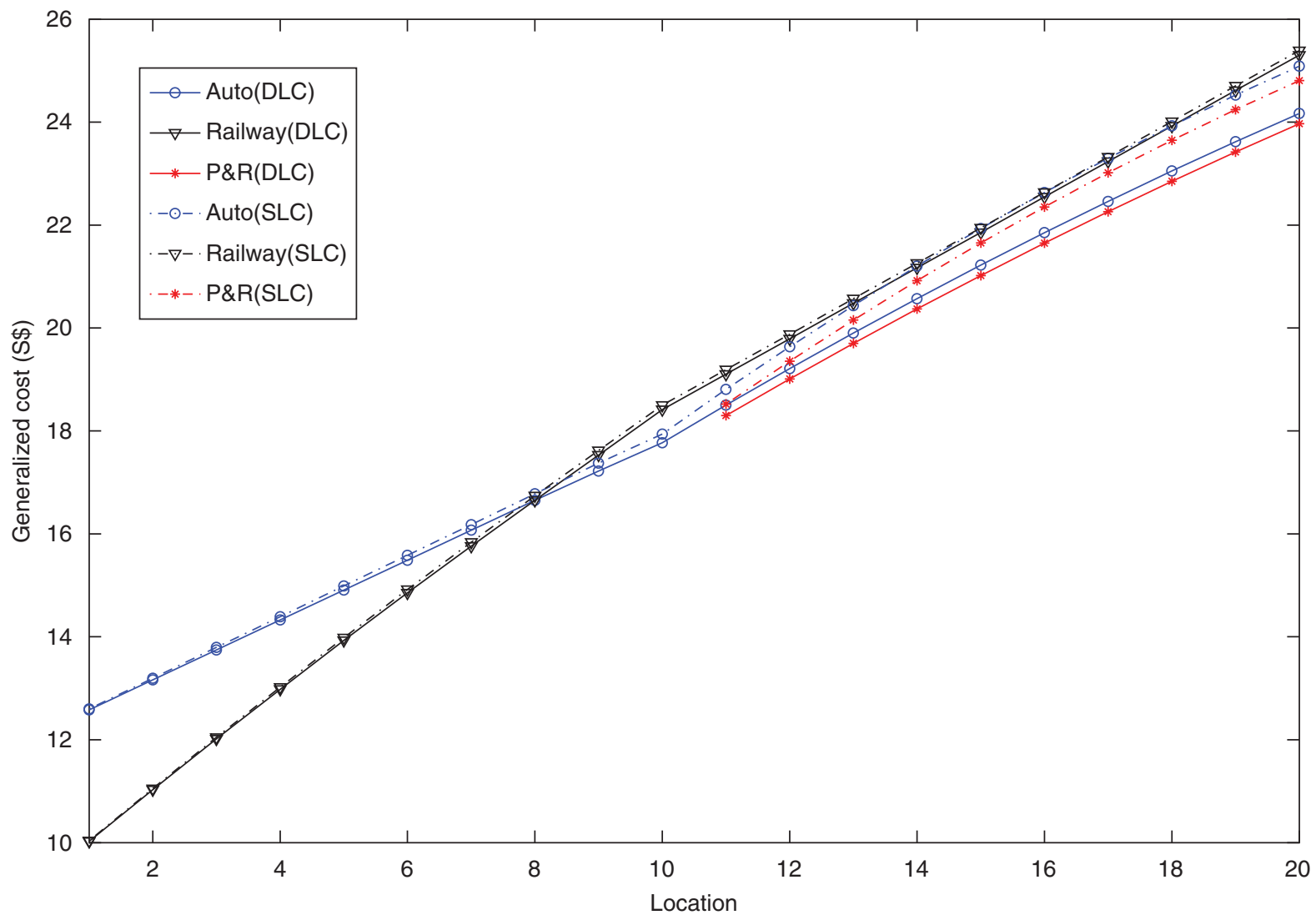

FIGURE 3 Comparison of generalized costs at equilibrium status between DLC and SLC.

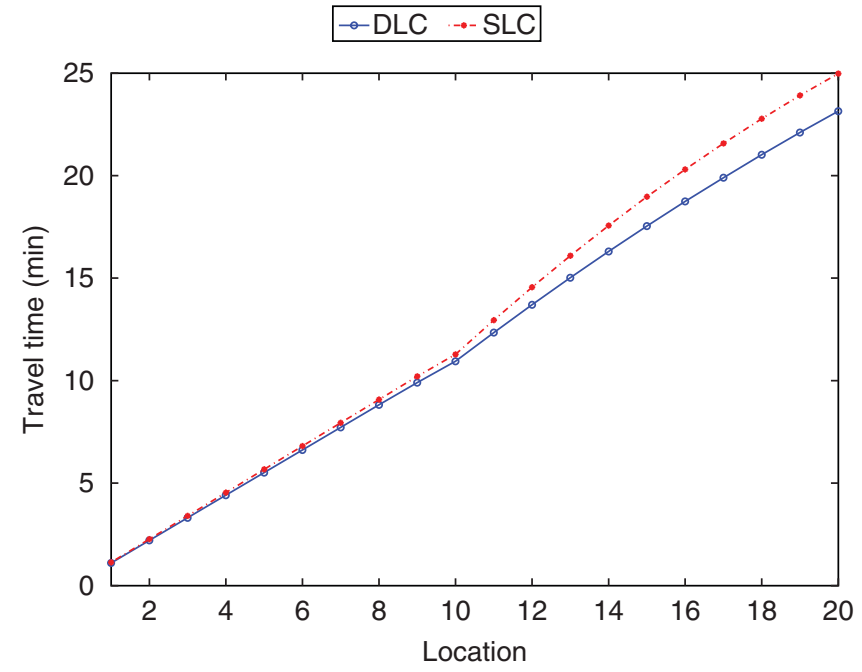

(a)

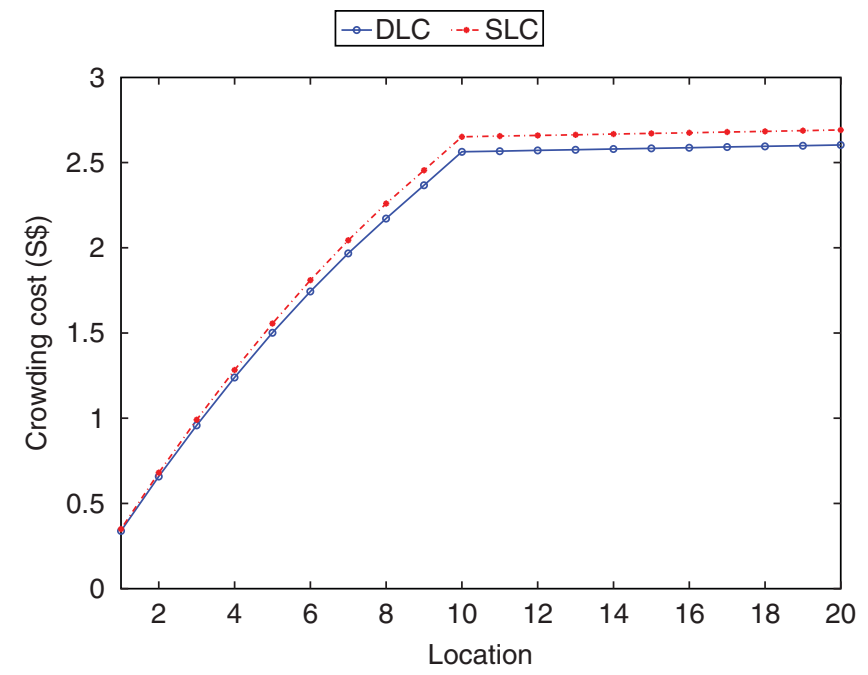

(b)

FIGURE 4 Comparison of travel time on highway and crowding cost on railway at equilibrium status between DLC and SLC: (a) travel time on highway and $(b)$ crowding cost on railway. 
TABLE 4 Total Generalized Costs Along Corridor at Equilibrium Status with P\&R Locations Considering SLC

\begin{tabular}{lc|ll}
\hline $\begin{array}{l}\text { P\&R Facility } \\
\text { Location } \\
\text { section) }\end{array}$ & $\begin{array}{l}\text { Total } \\
\text { Generalized } \\
\text { Cost }\end{array}$ & $\begin{array}{l}\text { P\&R Facility } \\
\text { Location } \\
\text { (section) }\end{array}$ & $\begin{array}{l}\text { Total } \\
\text { Generalized } \\
\text { Cost }\end{array}$ \\
\hline 1 & 2.9061 & 11 & 2.8668 \\
2 & 2.9061 & 12 & 2.8529 \\
3 & 2.9061 & 13 & 2.8516 \\
4 & 2.9061 & 14 & 2.8523 \\
5 & 2.9061 & 15 & 2.8567 \\
6 & 2.9061 & 16 & 2.8639 \\
7 & 2.9115 & 17 & 2.8729 \\
8 & 2.9227 & 18 & 2.8832 \\
9 & 2.9164 & 19 & 2.8946 \\
10 & 2.9081 & 20 & 2.9061 \\
\hline
\end{tabular}

NOTE: Cost values are in Singapore dollars $\left(1 \times 10^{5}\right)(1 \mathrm{~S} \$=0.81$ U.S in 2013).

after the SLC is considered, and the commuters' mode choice and $\mathrm{P} \& \mathrm{R}$ behaviors are characterized and analyzed. Numerical results show that public transit is preferred by the users living close to the $\mathrm{CBD}$, whereas the $\mathrm{P} \& \mathrm{R}$ mode is the choice for commuters far from the $\mathrm{CBD}$, and auto is also used around the middle part of the corridor, where the generalized cost of $P \& R$ is higher than the direct auto mode in terms of the high transfer cost of $P \& R$.

Application of the DVI modeling approach to the spatial equilibrium travel pattern with multimodal choices in a metropolitan area proved successful in this study. However, only the static equilibrium pattern is described here. In a future study, dynamic elements will be introduced to model a dynamic spatial equilibrium with multiple transportation modes in a traffic corridor.

\section{REFERENCES}

1. Noel, E. Park-and-Ride: Alive, Well, and Expanding in the United States. Journal of Urban Planning and Development, Vol. 114, No. 1, 1988, p. 2.
2. Verhoef, E., P. Nijkamp, and P. Rietveld. The Economics of Regulatory Parking Policies: The (IM)possibilities of Parking Policies in Traffic Regulation. Transportation Research Part A: Policy and Practice, Vol. 29, No. 2, 1995, pp. 141-156.

3. Niblette, R., and D. J. Palmer. Park-and-Ride in London and the South East. Journal of the Institution of Highway and Transportation, No. 40, 1993, pp. 4-10.

4. Foo, T. S. Experience from Singapore's Park-and-Ride Scheme (1975-1996). Habitat International, Vol. 21, No. 4, 1997, pp. 427-443.

5. Lam, W. H. K., N. M. Holyoak, and H. P. Lo. How Park-and-Ride Schemes Can Be Successful in Eastern Asia. Journal of Urban Planning D, ASCE, Vol. 127, No. 2, 2001, pp. 63-78.

6. Hounsell, N., B. Shrestha, and J. Piao. Enhancing Park and Ride with Access Control: A Case Study of Southampton. Transport Policy, Vol. 18, No. 1, 2011, pp. 194-203.

7. Garcia, R., and A. Marin. Parking Capacity and Pricing in Park'n Ride Trips: A Continuous Equilibrium Network Design Problem. Annals of Operations Research, Vol. 116, 2002, pp. 153-178.

8. Wang, J. Y. T., H. Yang, and R. Lindsey. Modelling Park-and-Ride Service in a Linear Monocentric City. Journal of the Eastern Asia Society for Transportation Studies, Vol. 5, 2003, pp. 1377-1392.

9. Wang, J. Y. T., H. Yang, and R. Lindsey. Locating and Pricing Park-andRide Facilities in a Linear Monocentric City with Deterministic Mode Choice. Transportation Research Part B: Methodological, Vol. 38, No. 8, 2004, pp. 709-731.

10. Liu, T. L., H. J. Huang, H. Yang, and X. N. Zhang. Continuum Modeling of Park-and-Ride Services in a Linear Monocentric City with Deterministic Mode Choice. Transportation Research Part B: Methodological, Vol. 43, No. 6, 2009, pp. 692-707.

11. Lo, H. K., X. W. Luo, and B. W. Y. Siu. Degradable Transport Network: Travel Time Budget of Travelers with Heterogeneous Risk Aversion. Transportation Research Part B: Methodological, Vol. 40, No. 9, 2006, pp. 792-806.

12. Pang, J. S., and D. Stewart. Differential Variational Inequalities. Mathematical Programming, Vol. 113, No. 2, 2008, pp. 345-424.

13. Lo, H. K., and Y. K. Tung. Network with Degradable Links: Capacity Analysis and Design. Transportation Research Part B: Methodological, Vol. 37, No. 4, 2003, pp. 345-363.

14. D'Este, G. Trip Assignment to Radial Major Roads. Transportation Research Part B: Methodological, Vol. 21, No. 6, 1987, pp. 433-442.

15. Huang, H. J. Fares and Tolls in a Competitive System with Transit and Highway: The Case with Two Groups of Commuters. Transportation Research Part E: Logistics and Transportation Review, Vol. 36, No. 4 2000, pp. 267-284.

16. Sherali, H. D., R. S. Krishnamurthy, and F. A. Al-Khayyal. Enumeration Approach for Linear Complementarity Problems Based on a Reformulation-Linearization Technique. Journal of Optimization Theory and Applications, Vol. 99, No. 2, 1998, pp. 481-507.

The Transportation Network Modeling Committee peer-reviewed this paper. 